Published in the special issue of Semiconductors in memory of V. I. Perel

Phys. Techn. Poluprovodn. 42, 1002 (2008) [Semiconductors 42, 989 (2008)]

\title{
Spin-orbit terms in multi-subband electron systems: A bridge between bulk and two-dimensional Hamiltonians
}

\author{
K.V. Kavokin? \\ A.F. Ioffe Physico-Technical Institute, 194021 St. Petersburg, Russia \\ M.E. Portno 团 \\ School of Physics, University of Exeter, Stocker Road, Exeter EX4 4QL, United Kingdom
}

(Dated: 24 June, 2008)

\begin{abstract}
We analyze the spin-orbit terms in multi-subband quasi-two-dimensional electron systems, and how they descend from the bulk Hamiltonian of the conduction band. Measurements of spin-orbit terms in one subband alone are shown to give incomplete information on the spin-orbit Hamiltonian of the system. They should be complemented by measurements of inter-subband spin-orbit matrix elements. Tuning electron energy levels with a quantizing magnetic field is proposed as an experimental approach to this problem.
\end{abstract}

PACS numbers: 73.21.Fg, 71.70.Ej, 73.90.+f

Spin-dependent phenomena in semiconductors was one of the favorite research themes for Vladimir Idelevich Perel' since the early 1970s. ${ }^{1}$ From the mid-1980s his interests shifted towards spin-related effects in low-dimensional systems, starting with optical orientation and polarization properties of hot photoluminescence in quantum-well structures, which was closely connected to experiments carried out by the group of D.N. Mirlin $\stackrel{2}{2}$ Some of this work was done together with one of us (MEP) $\stackrel{\underline{3}}{\underline{n}}$ In the series of more recent papers, the transition from the two-dimensional to quasi-three dimensional case was considered $\stackrel{\underline{4}}{=}$ Several latest publications of Vladimir Idelevich were focused on spindependent tunneling and the role played in it by spin-orbit interaction. $\underline{\underline{5}}^{\text {This }}$ has defined the subject choice for our contribution to the special issue devoted to his memory.

The spin-orbit interaction in semiconductors has been widely discussed recently in relation to some proposals of spin-electronic and quantum-computing devices. It is of considerable physical interest in itself, because due to strong gradients of atomic potentials within the crystal unit cell the spin-orbit terms in the effective-mass Hamiltonian are often greatly enhanced with respect to those of a free electron ${ }^{6}$ In addition, the reduced crystal symmetry brings about new spin-orbit terms unknown for free particles, the so-called Dresselhaus terms $\underline{\underline{\underline{T}}}$

In two-dimensional systems, spin-orbit effects are known to be even stronger than in bulk semiconductors. In particular, in the effective-mass two-dimensional (2D) Hamiltonian there appear spin-orbit terms which are linear in the 2D wave vector $\mathbf{k}$. They may exist in structures where the spatial inversion symmetry is broken. There are so-called bulk inversion asymmetry (BIA) terms, which appear on averaging the bulk Dresselhaus terms over the envelope function of the corresponding size-quantization level, and structure inversion asymmetry (SIA), or Rashba, terms,$\stackrel{8}{=}$ The latter are believed to exist in asymmetric quantum wells (QWs); there is plenty of experimental evidence of their existence in specific structures, but apparently no agreement has been reached as to how they descend from the bulk spin-orbit Hamiltonian. Some authors argue that they are entirely due to interfacial effects $\underline{\underline{9}}$ The most consistent theoretical treatment of this problem was carried out by Gerchikov and Subashiev $\underline{10}$ (see also a more recent paper by Winkler ${ }^{11}$ ). However, even these papers do not give explicit answers to questions arising when one attempts to devise experiments aimed at determination of spin-orbit parameters or to engineer structures with controllable spin-orbit effects.

In our opinion, for a full understanding of the spin-orbit effects in nanostructures it is necessary to take into consideration inter-subband spin-orbit coupling in multi-subband quantum-dimensional structures, which present a natural bridge between 3D and 2D semiconductor structures. In this paper we analyze the effect of the spin-orbit interaction on the energy spectrum of many-subband QWs, and show that applying a quantizing magnetic fields to such a system presents a way for experimental determination of all the relevant parameters of the spin-orbit interaction. Implications for optical and phonon spectroscopy are discussed.

We start with the phenomenological expression for the spin-orbit Hamiltonian of the conduction band of a compositionally homogeneous bulk semiconductor in the envelope-function approximation. It has the following general form: $\underline{6}^{-}$

$$
H_{S O}=\left(\mathbf{h}_{B I A} \cdot \mathbf{S}\right)+a_{V}([\mathbf{k} \times \nabla V] \cdot \mathbf{S}),
$$

where the "Dresselhaus field" $\mathbf{h}_{B I A}$, existing in non-centrosymmetric crystals, is a pseudovector that is an odd function 
of the components of the electron wave vector $\mathbf{k}$, and $V$ is the electrostatic potential energy. The second term in Eq. (11) has the same form as the spin-orbit Hamiltonian of a free electron; however, the constant $a_{V}$ is not equal to the vacuum spin-orbit constant $a_{v a c}=\hbar^{2} /\left(4 m_{e}^{2} c^{2}\right)$, where $m_{e}$ is the bare electron mass. As electrons in semiconductor crystals are actually subjected to strong potential gradients within the crystal unit cell, the spin-orbit interaction is enhanced by a factor of approximately $m_{e} c^{2} / E_{g}$, where $E_{g}$ is the semiconductor band gap. 12 This huge enhancement of the spin-orbit interaction has allowed, in particular, observation of spin-dependent currents due to anisotropic scattering of electrons by impurity centers (a solid-state analog of the Mott effect) in GaAs. $12,13,14$

In nanostructured semiconductors based on solid solutions like $\mathrm{Ga}_{x} \mathrm{Al}_{1-x} \mathrm{As}$, not only the electrostatic potential, but also the composition $x$ and, respectively, all the parameters of the band structure, may depend on the coordinates. One can therefore introduce a new phenomenological spin-orbit term proportional to $\nabla x$. Since all the band energies are, to the first approximation, linear in $x$, we shall write this term for electrons as $a_{X}\left(\left[\mathbf{k} \times \nabla E_{C}\right] \cdot \mathbf{S}\right)$. Here, $\nabla E_{C}$ is the "variable-gap" field that affects charge carriers in structures with gradients of composition. ${ }^{15}$

Using the standard $8 \times 8 \mathbf{~ k p}$ method of calculation of spin-orbit splitting, analoguos to that used in Refs. 9. 16, 17, 18, Gerchikov and Subashiev 10 have shown that the spin-orbit term in the conduction band Hamiltonian can be expressed through a gradient of an effective "spin-orbit potential" $\chi_{C}$ :

$$
H_{S O}=\left(\mathbf{h}_{B I A}+\left[\mathbf{k} \times \nabla \chi_{C}\right]\right) \cdot \mathbf{S}
$$

where $\chi_{C}$ is a function of the energy positions of the extrema of the conduction band $\left(\Gamma_{6}\right)$ and the uppermost valence bands $\left(\Gamma_{8}\right.$ and $\left.\Gamma_{7}\right)$ :

$$
\chi_{C}=\frac{P^{2} \Delta(x)}{3\left[E-E_{V}(x)\right]\left[E-E_{V}(x)+\Delta(x)\right]} .
$$

Here, $P$ is the momentum matrix element between $S$ and $P$ Bloch states, $E$ is the electron energy, $E_{V}(x)$ is the energy position of the top of the valence band, and $\Delta(x)$ is the spin-orbit energy splitting. As noted in Ref. 10, Eq. (2) is true even in interface regions, where $\nabla \chi_{C}$ can be expressed in terms of delta functions. In the following, we shall consider only structures where the energy of size quantization is much less than the bandgap $E_{g}$. This condition assumes that variations of both $V$ and $E_{C}$ are much less than $E_{g}$ over the region where size-quantized wave functions are mainly concentrated. This class of structures includes wide quantum wells or heterojunctions with high barriers, but in this case interfacial regions, where the electron probability density is small but the gradient of $E_{C}$ is very large, should be considered separately (see discussion below). For such structures, one can easily obtain from Eq (2) of Ref. 10 (compare also Eqs. (7) and (10) of Ref. 18) the following expressions for $a_{V}$ and $a_{X}$ :

$$
\begin{aligned}
& a_{V}=\frac{\hbar^{2} \Delta\left(2 E_{g}+\Delta\right)}{2 m E_{g}\left(E_{g}+\Delta\right)\left(3 E_{g}+2 \Delta\right)}, \\
& a_{X}=\frac{\hbar^{2} \Delta\left(2 E_{g}+\Delta\right)}{2 m E_{g}\left(E_{g}+\Delta\right)\left(3 E_{g}+2 \Delta\right)}\left[\frac{E_{g}^{2}}{\Delta\left(2 E_{g}+\Delta\right)} \frac{d \Delta}{d E_{C}}+\frac{d E_{V}}{d E_{C}}\right],
\end{aligned}
$$

where $m=3 \hbar^{2} P^{2} E_{g}\left(E_{g}+\Delta\right) / 2\left(E_{g}+2 \Delta\right)$ is the effective mass of the conduction-band electron. For example, using band parameters and their dependence on composition in $\mathrm{Ga}_{1-x} \mathrm{Al}_{x}$ As from Ref. 19 , one obtains $a_{V}=4.1 \times 10^{-16}$ $\mathrm{cm}^{-2}$, and $a_{X}=-3.1 \times 10^{-16} \mathrm{~cm}^{-2}$ near $x=0$. For the class of structures we consider, spin-orbit constants, as well as the effective mass, can be approximately treated as spatially invariable parameters.

We can now analyze how bulk spin-orbit terms transform as a result of size quantization in QWs. Due to translational invariance in the QW plane, the general form of the matrix element of the spin-orbit Hamiltonian between electron eigenfunctions in the QW is:

$$
\left\langle m \mathbf{k}_{1} \mu\left|\hat{H}_{S O}\right| n \mathbf{k}_{2} \nu\right\rangle=\left(\mathbf{h}_{m n}(\mathbf{k}) \cdot \mathbf{S}\right)_{\mu \nu} \delta\left(\mathbf{k}-\mathbf{k}_{1}\right) \delta\left(\mathbf{k}-\mathbf{k}_{2}\right)
$$

where $m$ and $n$ enumerate size-quantization subbands, $\mathbf{k}_{1}$ and $\mathbf{k}_{2}$ are electron wave vectors, and $\mu$ and $\nu$ are spin indices.

Let us first consider the SIA (Rashba) terms. If all the potential gradients are normal to the QW plane (we denote the corresponding unit vector as $\mathbf{n})$, the spin-orbit field $\mathbf{h}_{m n}(\mathbf{k})$ takes the form:

$$
\mathbf{h}_{m n}^{S I A}(\mathbf{k})=A_{m n}^{S I A}[\mathbf{n} \times \mathbf{k}]
$$


where

$$
\begin{aligned}
A_{m n}^{S I A} & =\int_{-\infty}^{+\infty} \Psi_{m}(z) \Psi_{n}(z) \frac{d \chi_{C}}{d z} d z \\
& =a_{V} \int_{-\infty}^{+\infty} \Psi_{m}(z) \Psi_{n}(z) \frac{d V}{d z} d z+a_{X} \int_{-\infty}^{+\infty} \Psi_{m}(z) \Psi_{n}(z) \frac{d E_{C}}{d z} d z+\sum_{i} I_{m n}^{i} .
\end{aligned}
$$

Here, $\Psi_{m}(z)$ and $\Psi_{n}(z)$ are envelope functions of the $m$ th and $n$th subband in the $\mathbf{z}$-direction, respectively. The values of $a_{V}$ and $a_{X}$ correspond to the bottom of the QW. The last term is an interfacial contribution, which arises because at interfaces sharp gradients of $E_{C}$ coincide with abrupt changes of $\chi_{C}$; summation is taken over all the interfaces. Since wave functions are continuous at interfaces, the overall contribution of interface regions to the matrix element $A_{m n}^{S I A}$ can be written as $\Psi_{m}\left(z_{i}\right) \Psi_{n}\left(z_{i}\right)\left(\chi_{C}\left(z_{i+}\right)-\chi_{C}\left(z_{i-}\right)\right)$, where $z_{i+}$ and $z_{i-}$ denote positions immediately to the right and to the left of the interface, respectively. The term $I_{m n}^{i}$ is therefore equal to the difference between this expression and the contribution of the interface to the integral in the second term of Eq. (7):

$$
\begin{aligned}
I_{m n}^{i} & =\Psi_{m}\left(z_{i}\right) \Psi_{n}\left(z_{i}\right)\left[\chi_{C}\left(z_{i+}\right)-\chi_{C}\left(z_{i-}\right)\right]-\Psi_{m}\left(z_{i}\right) \Psi_{n}\left(z_{i}\right) a_{X}\left[E_{C}\left(z_{i+}\right)-E_{C}\left(z_{i-}\right)\right]= \\
& =\Psi_{m}\left(z_{i}\right) \Psi_{n}\left(z_{i}\right)\left(\bar{a}_{i}-a_{X}\right)\left[E_{C}\left(z_{i+}\right)-E_{C}\left(z_{i-}\right)\right],
\end{aligned}
$$

where $\bar{a}_{i}=\left[\chi_{C}\left(z_{i+}\right)-\chi_{C}\left(z_{i-}\right)\right] /\left[E_{C}\left(z_{i+}\right)-E_{C}\left(z_{i-}\right)\right]$ has the meaning of an effective constant $a_{X}$, renormalized due to abrupt changes of the band structure at the interface. For example, for the $\mathrm{GaAs} / \mathrm{Ga}_{0.6} \mathrm{Al}_{0.4} \mathrm{As}$ interface, $\bar{a}_{i}=-2.5 \times 10^{-16} \mathrm{~cm}^{2}$. Since the bulk constant $a_{X}=-3.1 \times 10^{-16} \mathrm{~cm}^{2}$, the difference $\left|\bar{a}_{i}-a_{X}\right|$ is much smaller than $\left|a_{X}\right|$, and we can conclude that the interfacial corrections are not significant in the $\mathrm{GaAs} / \mathrm{Ga}_{1-x} \mathrm{Al}_{x} \mathrm{As}$ system.

As the QW localization potential for electrons is $U(z)=V(z)+E_{C}(z)$, Eq. (7) can be rewritten in the following way:

$$
A_{m n}^{S I A}=a_{X} \int_{-\infty}^{+\infty} \Psi_{m}(z) \Psi_{n}(z) \frac{d U}{d z} d z+\left(a_{V}-a_{X}\right) \int_{-\infty}^{+\infty} \Psi_{m}(z) \Psi_{n}(z) \frac{d V}{d z} d z+\sum_{i} I_{m n}^{i} .
$$

Making use of the fact that $\frac{d U}{d z}=-\dot{p}_{z}$, where $p_{z}$ is the $z$-component of the electron momentum, we obtain: $:^{20}$

$$
\int_{-\infty}^{+\infty} \Psi_{m}(z) \Psi_{n}(z) \frac{d U}{d z} d z=-\frac{i}{\hbar}\left(E_{n}-E_{m}\right) \int_{-\infty}^{+\infty} \Psi_{m}(z) \hat{p}_{z} \Psi_{n}(z) d z=\left(E_{m}-E_{n}\right) \int_{-\infty}^{+\infty} \Psi_{m}(z) \frac{d}{d z} \Psi_{n}(z) d z,
$$

where $E_{n}$ and $E_{m}$ are energy levels corresponding to eigenfunctions $\Psi_{n}(z)$ and $\Psi_{m}(z)$.

Considering the second term in Eq. (9), we recall that $\mathcal{E}=-(1 / e) /(d V / d z)$ is an electric field which is constant across the quantum well unless there are electric charges inside the well. Modern nanostructure technology usually avoids placing impurity atoms inside the quantum well, so an inhomogeneity of $\mathcal{E}$ may normally arise only due to screening by the electron gas in QWs containing free electrons. If the concentration of two-dimensional electrons is small, the electric field inhomogeneity can be neglected. For a constant $\mathcal{E}, \int_{-\infty}^{+\infty} \Psi_{m}(z) \Psi_{n}(z) \mathcal{E} d z=\mathcal{E} \delta_{m n}$. In this case, we come to the following expression for $A_{m n}^{S I A}$ :

$$
A_{m n}^{S I A}=a_{X}\left(E_{m}-E_{n}\right) \int_{-\infty}^{+\infty} \Psi_{m}(z) \frac{d}{d z} \Psi_{n}(z) d z-\left(a_{V}-a_{X}\right) e \mathcal{E} \delta_{m n}+\sum_{i} I_{m n}^{i} .
$$

The value $A_{n n}^{S I A}=-\left(a_{V}-a_{X}\right) e \mathcal{E}+\sum_{i} I_{n n}^{i}$ is the coefficient of the Rashba term in the $n$th subband. One can see that, contrary to the widespread opinion, the Rashba term is not entirely due to interfacial effects; in fact, simple estimations show that the interfacial term is much smaller than the bulk contribution proportional to the electric field and to the difference of the spin-orbit constants $a_{V}$ and $a_{X}$. A parabolic quantum well formed by modulation of composition is a good example illustrating this property of SIA spin-orbit terms. There are no interfaces in the parabolic quantum well. Moreover, applying an electric field in this case does not change the shape of the localizing potential, which remains symmetric:

$$
U(z)=\frac{m \omega^{2} z^{2}}{2}-\mathcal{E} e z=\frac{m \omega^{2}}{2}\left(z-z_{0}\right)^{2}-\frac{m \omega^{2} z_{0}^{2}}{2},
$$


where $z_{0}=\mathcal{E} e / m \omega^{2}$. However, according to Eq. (11), there exist both intra- and intersubband SIA terms:

$$
\begin{aligned}
A_{n-1, n}^{S I A} & =A_{n, n-1}^{S I A}=a_{X} \sqrt{\frac{m \hbar \omega^{3} n}{2}}, \\
A_{n, n}^{S I A} & =-\left(a_{V}-a_{X}\right) \mathcal{E} .
\end{aligned}
$$

Intra-subband SIA terms are zero in the absence of electric fields, while inter-subband SIA matrix elements are always present and do not depend on the electric field. Remarkably, bandgap gradients do not contribute to the Rashba term: one needs to apply an electric field to the structure to produce it. These gradients are needed, however, to confine the electron in the $\mathbf{z}$-direction; if the electron was confined by a purely electrostatic potential, Rashba terms would also be absent. This fact has been noted by Gerchikov and Subashiev $\underline{10}$

Another instructive example is a symmetric rectangular quantum well in an electric field. Since in this case all the changes of composition $x$ are concentrated at interfaces, the interfacial effects can be exactly accounted for by replacing $a_{X}$ with $\bar{a}_{i}$ in Eq. (11); this substitution eliminates the interfacial term. One can see that in $\mathrm{GaAs} / \mathrm{Ga}_{0.6} \mathrm{Al}_{0.4} \mathrm{As}$ structures the interfacial correction amounts to less than $10 \%$ of the intrasubband Rashba term, and about $20 \%$ of the intersubband SIA matrix element.

For a single heterojunction and other structures where the electric field is created by separated charges of impurities in depleted doped regions and the 2D electron gas itself, Eq. (11) should be modified by taking into account the electric field inhomogeneity. In this case, the electric field $\mathcal{E}$ in the Rashba term should be replaced with its average value (integrated with the squared wave function of the corresponding subband). The field inhomogeneity will also contribute to the inter-subband term. The correponding corrections can be evaluated using the Poisson equation. For example, the leading correction to the first term in Eq.(11) is: $\tilde{A}_{m n}^{S I A}=\left(a_{V}-a_{X}\right)\left(4 \pi n_{s} e / \varepsilon\right) \int_{-\infty}^{+\infty} \Psi_{m}(z) \Psi_{n}(z) \int_{-\infty}^{z} \Psi_{0}^{2}\left(z^{\prime}\right) d z^{\prime} d z$, where $m \neq n, n_{s}$ is the sheet concentration of electrons, $e$ is the absolute value of the electron charge, $\varepsilon$ is the dielectric constant, and $\Psi_{0}^{2}$ is the squared ground-state wave function (here we assume that most electrons are in the lowest size-quantization subband; otherwise summation over all occupied subbands should be performed).

BIA (Dresselhaus) terms in bulk zinc-blende semiconductors have the form $\hat{H}_{B I A}=\mathbf{h}^{\text {bulk }}(\mathbf{k}) \cdot \mathbf{S}$, with

$$
h_{x}^{b u l k}=\alpha \hbar^{3}\left(m_{e} \sqrt{2 m_{e} E_{g}}\right)^{-1} k_{x}\left(k_{y}^{2}-k_{z}^{2}\right),
$$

where $m_{e}$ is the effective mass of the electron, $E_{g}$ is the band gap, and $k_{x}, k_{y}, k_{z}$ are components of the wave vector along the cubic axes [100], [010], and [001] respectively. The $\mathbf{y}$ - and $\mathbf{z}$-components of $\mathbf{h}^{\text {bulk }}$ are obtained from Eq. (14) by permutation of indices.

The expression for the BIA matrix elements between 2D subbands depends on the QW orientation with respect to crystal axes. For the most common case of a [100] quantum well

$$
\begin{aligned}
\mathbf{h}_{m n}^{\mathrm{BIA}} & =A_{m n}^{\mathrm{BIA}}\left(k_{x} \mathbf{e}_{x}-k_{y} \mathbf{e}_{y}\right)+i \frac{\alpha \hbar^{3}}{m \sqrt{2 m E_{g}}} \\
& \times \int_{-\infty}^{+\infty} \Psi_{m}(z) \frac{d}{d z} \Psi_{n}(z) d z\left(k_{x}^{2}-k_{y}^{2}\right) \mathbf{e}_{z},
\end{aligned}
$$

where

$$
A_{m n}^{\mathrm{BIA}}=\frac{\alpha \hbar^{3}}{m \sqrt{2 m E_{g}}} \int_{-\infty}^{+\infty} \Psi_{m}(z) \frac{d^{2}}{d z^{2}} \Psi_{n}(z) d z .
$$

A comparison of Eqs. (11) and (15) shows that BIA and SIA terms in quantum wells are related to the corresponding bulk Hamiltonians in different ways. Intra-subband BIA terms are determined by a unique constant $\alpha$, the same as in bulk BIA terms. This is confirmed experimentally: the values of $\alpha$ measured from spin relaxation in bulk

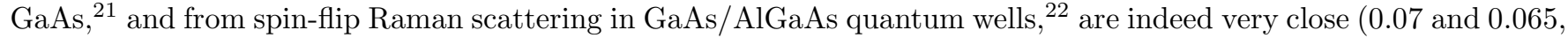
respectively). On the contrary, Rashba terms depend on a specific combination of constants $\left(a_{V}-a_{X}\right)$ describing different contributions from gradients of electrostatic and crystal potentials, while, for instance, only $a_{V}$ contributes to spin-dependent scattering from charged impurities in the bulk. These constants cannot be determined separately, only by measuring spin-orbit splitting in size-quantization subbands. These measurements do not, therefore, give complete information about the spin-orbit Hamiltonian of the conduction band. 
Experimental determination of inter-subband spin-flip matrix elements, which would be very helpful in this view, is hindered by the fact that they are typically much smaller than the energy of size quantization, and all the observable effects of these matrix elements are consequently suppressed. We should like to note that this difficulty can, in principle, be overcome by tuning energy levels of quasi-2D electrons with a strong magnetic field. When a magnetic field is applied normal to the QW plane, the in-plane motion of electrons is also quantized, and the continuum energy spectrum of each 2D subband is transformed into discrete Landau levels (LLs). Landau levels belonging to different subbands $n$ and $m$ can be tuned in resonance by choosing the magnetic field so that $\left|E_{n}-E_{m}\right|=l \hbar \omega_{c}$, where $l$ is an integer, and $\omega_{c}$ is the cyclotron frequency. Under these conditions, the energy spectrum and the structure of wave functions of the four-level subsystem formed by Zeeman-split spin components of the two LLs from different subbands are expected to be strongly affected by the spin-orbit interaction.

Using the Landau gauge $\left(A_{x}=-H y ; A_{y}=A_{z}=0\right)$ and Eqs. (5), (6), (11), (15) and (16), we obtain the following expressions for matrix elements between electron states differing by subband number, LL number, and spin projection on the normal to the structure plane:

$$
\begin{gathered}
\left\langle\eta, n, k_{x},-1 / 2\left|H_{S O}\right| \mu, n-1, k_{x},+1 / 2\right\rangle=i A_{\eta \mu}^{S I A} \frac{1}{l_{H}} \sqrt{2 n}, \\
\left\langle\eta, n-1, k_{x},+1 / 2\left|H_{S O}\right| \mu, n, k_{x},-1 / 2\right\rangle=-i A_{\eta \mu}^{S I A} \frac{1}{l_{H}} \sqrt{2 n} \\
\left\langle\eta, n, k_{x},+1 / 2\left|H_{S O}\right| \mu, n-1, k_{x},-1 / 2\right\rangle=A_{\eta \mu}^{B I A} \frac{1}{l_{H}} \sqrt{2 n} \\
\left\langle\eta, n-1, k_{x},-1 / 2\left|H_{S O}\right| \mu, n, k_{x},+1 / 2\right\rangle=A_{\eta \mu}^{B I A} \frac{1}{l_{H}} \sqrt{2 n}
\end{gathered}
$$

where indices $\eta$ and $\mu$ enumerate subbands, $n$ is the LL number, and $l_{H}$ is the magnetic length. Remarkably, states from different subbands and LLs are coupled by either BIA or SIA terms, but not by the two types of spin-orbit terms at the same time: Eqs. (17) and (18) couple states $\langle n-1,+1 / 2|$ and $\langle n,-1 / 2|$, while Eqs. (19) and (20) couple states $\langle n-1,-1 / 2|$ and $\langle n,+1 / 2|$. Therefore, by tuning a specific pair of levels into resonance one can prepare an effective two-level system whose spectrum is determined by either the BIA or SIA term. As Eqs. (17)-(20) are based on general symmetry properties of Rashba and Dresselhaus terms rather than on any specific method of derivation of corresponding constants, they can be used for experimental determination of spin-orbit parameters.

The exact form of this spectrum and its experimental manifestations depend on the electron concentration in the quasi-2D system. If the concentration is low and many-body effects are absent, the inter-subband spin-orbit coupling results in anticrossing of the two levels. The resulting energy gaps, equal to corresponding inter-subband matrix elements (Eqs. (17)-(20)) can be measured by high-resolution spectroscopic techniques (optical or spin/cyclotron resonance spectroscopy), as has been proposed for one-subband systems in tilted magnetic fields. 23 The admixture of wave functions with opposite spin near the anticrossing can, in principle, be detected using optical polarization spectroscopy. If all the electron states below the two selected levels are filled, many-body effects have been shown to strongly affect the spectrum of electrons. In particular, they result in the opening of gaps in the energy spectrum even in the absence of spin-orbit interaction ${ }^{24}$ It has been proposed to employ non-equilibrium phonons to probe

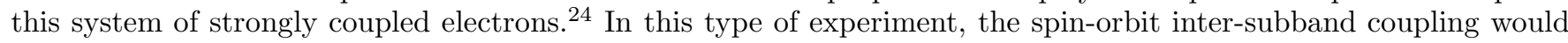
manifest itself by allowing phonon-assisted transitions between levels with different projections of the electron spin, which are otherwise forbidden ${ }^{24,25}$.

In conclusion, we have analyzed the transition from bulk to two-dimensional behavior of the spin-orbit interaction in multi-subband quasi-two-dimensional heterostructures. Intra- and inter-subband matrix elements of the spin-orbit Hamiltonian are derived, and the role of interfacial effects is estimated. The two types of spin-orbit terms (Rashba and Dresselhaus) can be distinguished by measuring the inter-subband coupling of Landau levels in quantizing magnetic fields.

We are grateful to the Editors for inviting us to submit a paper to the special issue devoted to the memory of V.I. Perel'. His work and unique personality strongly influenced our research and lit up our lives.

\footnotetext{
* E-mail: kidd.orient@mail.ioffe.ru
} 
† E-mail: m.e.portnoi@exeter.ac.uk

1 M.I. Dyakonov and V.I. Perel, JETP Lett. 13, 144 (1971); M.I. Dyakonov and V.I. Perel, JETP Lett. 13, 467 (1971); M.I. Dyakonov and V.I. Perel, Phys. Lett. A 35, 459 (1971); M.I. Dyakonov and V.I. Perel, Sov. Phys. JETP 33, 1053 (1971); for the review of early work see Optical Orientation, edited by F. Meier and B.P. Zakharchenya (North-Holland, Amsterdam, 1984; Nauka, Leningrad, 1989).

2 D.N. Mirlin and V.I. Perel, Semicond. Sc. Technol. 7, 1221 (1992).

3 I.A. Merkulov, V.I. Perel and M.E. Portnoi, Zh. Exp. Teor. Fiz. 99, 1202, 1991 [Sov. Phys. JETP 72, 669 (1991)]; I.A. Merkulov, V.I. Perel and M.E. Portnoi, Supperlatt. Microstruct. 10, 371 (1991), V.I. Perel' and M.E. Portnoi, Fiz. Tekh. Poluprovodn. 26, 2112 (1992) [Sov. Phys. Semicond. 26, 1185 (1992)].

4 V.F. Sapega, V.I. Perel, A.Yu. Dobin, D.N. Mirlin, I.A. Akimov, T. Ruf, M. Cardona, K. Eberl. Phys. Rev. B 56, 6871 (1997); V.F. Sapega, V.I. Perel', D.N. Mirlin, I.A. Akimov, T. Ruf, M. Cardona, W. Winter, and K. Eberl, Fiz. Tekh. Poluprovodn. 33, 738 (1999) [Semiconductors 33, 681 (1999)]; I.A. Akimov, D.N. Mirlin, V.I. Perel', and V.F. Sapega, Fiz. Tekh. Poluprovodn. 35, 758 (2001) [Semiconductors 35, 727 (2001)].

5 V.I. Perel', S.A. Tarasenko, I.N. Yassievich, S.D. Ganichev, V.V. Bel'kov, and W. Prettl, Phys. Rev. B 67, 201304 (2003); I.N. Yassievich and V.I. Perel, Physica B 340, 496 (2003); S.A. Tarasenko, V.I. Perel', and I.N. Yassievich, Phys. Rev. Lett. 93, 056601 (2004).

${ }^{6}$ G.L. Bir and G.E. Pikus, Symmetry and Strain-induced Effects in Semiconductors (Wiley, New York, 1974), Secs. 25 and 26.

7 G. Dresselhaus, Phys. Rev. 100, 580 (1955).

8 E.I. Rashba, Fiz. Tv. Tela (Leningrad) 2, 1224 (1960) [Sov. Phys. Solid State 2, 1109 (1960)]; Yu.A. Bychkov and E.I. Rashba, Usp. Fiz. Nauk 146, 531 (1985) [Sov. Phys. Usp. 28, 632 (1985)].

${ }^{9}$ P. Pfeffer and W. Zawadzki, Phys. Rev. B 59, R5312 (1999).

10 L.G. Gerchikov and A. V. Subashiev, Fiz. Tekh. Poluprovodn. 26, 131 (1992) [Sov. Phys. Semicond. 26, 73 (1992)].

11 R. Winkler, Physica E 22, 450 (2004).

12 V.N. Abakumov, V.V. Akulinichev, and I.N. Yassievich, Fiz. Tekh. Poluprovodn. 9, 936 (1975) [Sov. Phys. Semicond. 9, $612(1975)]$.

13 N.S. Averkiev and M.I. D'yakonov, Fiz. Tekh. Poluprovodn. 17, 629 (1983) [Sov. Phys. Semicond. 17, 393 (1983)].

14 A.A. Bakun, B.P. Zakharchenya, A.A. Rogachev, M.N. Tkachuk, and V.G. Fleisher, Pis'ma Zh. Eksp. Teor. Fiz. 40, 464 (1984) [JETP Lett. 40, 1293 (1984)].

15 A.S. Volkov, A.L. Lipko, Sh.M. Meretliev, and B.V. Tsarenkov, Pis'ma Zh. Eksp. Teor. Fiz. 41, 458 (1985) [JETP Lett. 41, 557 (1985)].

16 F.J. Ohkawa and Y. Uemura, J. Phys. Soc. Jpn. 37, 1325 (1974).

17 R. Lassnig, Phys. Rev. B 31, 8076 (1985).

18 E.A. de Andrada e Silva, G.C. La Rocca, and F. Bassani, Phys. Rev. B 50, 8523 (1994).

19 Physics of Group IV Elements and III-V Compounds, edited by O. Madelung, Landolt-Börnstein: Numerical Data and Functional Relationships in Science and Technology (Springer-Verlag, Berlin, 1982), Vol. 17, Subvolume A.

20 L.D. Landau and E.M. Lifshitz, Quantum Mechanics (Pergamon Press, New York, 1977).

21 Optical Orientation, edited by F. Meier and B.P. Zakharchenya, Modern Problems in Condensed Matter Sciences (NorthHolland, Amsterdam, 1984), Vol. 8.

${ }^{22}$ B. Jusserand, D. Richards, G. Allan, C. Priester, and B. Etienne, Phys. Rev. B 51, R4707 (1995).

23 V.I. Falko, Phys. Rev. B 46, R4320 (1992).

24 V.M. Apalkov and M.E. Portnoi, Phys. Rev. B 65, 125310 (2002); V.M. Apalkov and M.E. Portnoi, Physica E 15, 202 (2002).

25 V.N. Golovach and M.E. Portnoi, Phys. Rev. B 74, 085321 (2006). 